\title{
The study of gravitational collapse model in higher dimensional space-time
}

\author{
Ujjal Debnath* and Subenoy Chakraborty \\ Department of Mathematics, Jadavpur University, Calcutta-32, India.
}

(Dated: June 10, 2018)

\begin{abstract}
We investigate the end state of the gravitational collapse of an inhomogeneous dust cloud in higher dimensional space-time. The naked singularities are shown to be developing as the final outcome of non-marginally bound collapse. The naked singularities are found to be gravitationally strong in the sense of Tipler.

PACS numbers: 04.20.Dw
\end{abstract}

The gravitation collapse is an important and challenging issue in Einstein gravity, especially after the formation of famous singularity theorems [1] and Cosmic Censorship Conjecture (CCC) [2]. Also from the point of view of black hole physics and its astrophysical implications, it is interesting to know the final fate of gravitational collapse [3] in the background of general relativity. The singularity theorems as such can not predict about the visibility of the singularity to an external observer as well as their strength. On the other hand, the CCC is incomplete $[4,5]$ in the sense that there is no formal proof of it as well as there are counter examples of it. However, it has been pointed out recently [6] that the nature of the central shell focusing singularity depends on the choice of the initial data.

The study of higher dimensional gravitational collapse (in Tolman-Bondi form) was originated by Banerjee etal [7] and subsequently by Ghosh and Beesham [8] but they restricted to only self-similar and non self-similar solutions for marginally bound case. Recently, Ghosh and Banerjee [9] have studied naked singularity for five dimensional Tolman-Bondi model for self-similar solution in non-marginally bound case.

Moreover recently, Joshi etal [10] have shown that physically shear responsible for the formation of naked singularity. Subsequently Banerjee etal[11] and Debnath and Chakraborty [12] have studied gravitational collapse in higher dimensional Tolman-Bondi model for both marginally bound and non marginally bound cases. They have interestingly shown that for marginally bound case naked singularity is possible only upto five dimension while naked singularity may be possible in all dimensions for non marginally bound case. Then Goswami and Joshi [13] have pointed out that this peculiar feature of naked singularity (in marginal bound case) is due to the choice of the initial condition.

In the present paper we study gravitational collapse for non-marginally bound case considering non self-similar solutions and it is generalization to higher dimension of the Dwivedi and Joshi [14]. The geodesic equations can not completely solved due to the presence of the complicated hypergeometric function. So we present numerical results which favour the formation of naked singularity in any dimension.

The $n$ dimensional Tolman-Bondi metric in co-moving co-ordinates is given by

$$
d s^{2}=e^{\nu} d t^{2}-e^{\lambda} d r^{2}-R^{2} d \Omega_{n-2}^{2}
$$

where $\nu, \lambda, R$ are functions of the radial co-ordinate $r$ and time $t$ and $d \Omega_{n-2}^{2}$ represents the metric on the $(n-2)$-sphere. Since we assume the matter in the form of dust, the motion of particles will be geodesic allowing us to write $e^{\nu}=1$. Now from the Einstein's field equations for the metric (1), one can obtain

*Electronic address: ujjaldebnath@yahoo.com

†Electronic address: subenoyc@yahoo.co.in 


$$
e^{\lambda}=\frac{R^{\prime 2}}{1+f(r)}
$$

and

$$
\dot{R}^{2}=f(r)+\frac{F(r)}{R^{n-3}},
$$

where, $f(r)$ and $F(r)$ are arbitrary functions of radial co-ordinate $r$ alone with the restriction $1+f(r)>0$ for obvious reasons. Physically $F(r)$ and $f(r)$ represents the mass function and the binding energy function respectively.

The energy density $\rho(t, r)$ is therefore given by

$$
\rho(t, r)=\frac{(n-2) F^{\prime}(r)}{2 R^{n-2} R^{\prime}}
$$

Since in the present discussion we are concerned with the gravitational collapse, we require that $\dot{R}(t, r)<0$. As it is possible to make an arbitrary relabeling of spherical dust shells by $r \rightarrow g(r)$ without loss of generality, we fix the labeling by requiring that on the hypersurface $t=0, r$ coincides with the radius

$$
R(0, r)=r
$$

So the initial density is given by

$$
\rho(r) \equiv \rho(0, r)=\frac{n-2}{2} r^{2-n} F^{\prime}(r) \Rightarrow F(r)=\frac{2}{n-2} \int \rho(r) r^{n-2} d r
$$

Now from eq.(4) it can be seen that the density diverges faster in 5D $(n=5)$ as compared to $4 \mathrm{D}(n=4)$. For increasing dimensions the density diverges rapidly. Hence there is relatively more mass-energy collapsing in the higher dimensional space-time compared to the 4D and 5D cases.

Integrating eq.(3) and using the relation (5), we have the solution

$$
t=\frac{2}{(n-1) \sqrt{F}}\left[r^{\frac{n-1}{2}}{ }_{2} F_{1}\left[\frac{1}{2}, a, a+1,-\frac{f r^{n-3}}{F}\right]-R^{\frac{n-1}{2}}{ }_{2} F_{1}\left[\frac{1}{2}, a, a+1,-\frac{f R^{n-3}}{F}\right]\right]
$$

where ${ }_{2} F_{1}$ is the usual hypergeometric function with $a=\frac{1}{2}+\frac{1}{n-3}$.

Let us assume [14]

$$
\left.\begin{array}{l}
F(r)=r^{n-3} \lambda(r) \\
\alpha=\alpha(r)=\frac{r f^{\prime}}{f} \\
\beta=\beta(r)=\frac{r F^{\prime}}{F} \\
R(t, r)=r P(t, r)
\end{array}\right\}
$$

So using equations (3), (7) and (8), we have the following expressions 


$$
\begin{gathered}
R^{\prime}=\frac{1}{n-3}\left[P(\beta-\alpha)+\frac{1}{n-1}\left\{P_{2} F_{1}\left[\frac{1}{2}, a, a+1,-\frac{f P^{n-3}}{\lambda}\right]-P^{\frac{3-n}{2}}{ }_{2} F_{1}\left[\frac{1}{2}, a, a+1,-\frac{f}{\lambda}\right]\right\}\right. \\
\left.\{\alpha(n-1)-2 \beta\} \sqrt{1+\frac{f P^{n-3}}{\lambda}}+\frac{(\alpha-\beta+n-3) P^{\frac{3-n}{2}} \sqrt{1+\frac{f P^{n-3}}{\lambda}}}{\sqrt{1+\frac{f}{\lambda}}}\right]
\end{gathered}
$$

and

$$
\begin{array}{r}
\dot{R}^{\prime}=\frac{1}{r}\left[\frac{1}{n-1}\left\{{ }_{2} F_{1}\left[\frac{1}{2}, a, a+1,-\frac{f P^{n-3}}{\lambda}\right]-P^{\frac{1-n}{2}}{ }_{2} F_{1}\left[\frac{1}{2}, a, a+1,-\frac{f}{\lambda}\right]\right\}\{\alpha(n-1)-2 \beta\}\right. \\
\left.-\alpha \sqrt{1+\frac{f P^{n-3}}{\lambda}}+\frac{(\alpha-\beta+n-3) P^{\frac{1-n}{2}}}{\sqrt{1+\frac{f}{\lambda}}}\right]
\end{array}
$$

When $\lambda(r)=$ constant and $f(r)=$ constant, the space-time becomes self-similar. Now we restrict ourselves to functions $f(r)$ and $\lambda(r)$ which are analytic at $r=0$, such that $\lambda(0)>0$. From eq.(4) it can be seen that the density at the centre $(r=0)$ is finite at any time $t$, but becomes singular at $t=0$.

We wish to investigate whether the singularity, when the central shell with co-moving coordinate $(r=0)$ collapses to the centre at the time $t=0$, is naked . The singularity is naked if there exists a null geodesic that emanates from the singularity. Let $K^{a}=\frac{d x^{a}}{d \mu}$ be the tangent vector to the radial null geodesic, where $\mu$ is the affine parameter. Then we derive the following equations:

$$
\begin{gathered}
\frac{d K^{t}}{d \mu}+\frac{\dot{R}^{\prime}}{\sqrt{1+f}} K^{r} K^{t}=0 \\
\frac{d t}{d r}=\frac{K^{t}}{K^{r}}=\frac{R^{\prime}}{\sqrt{1+f(r)}}
\end{gathered}
$$

Let us define $X=\frac{t}{r}$, so that $P(t, r)=P(X, r)$. So eq.(7) becomes

$$
X=\frac{2}{(n-1) \sqrt{\lambda}}\left\{{ }_{2} F_{1}\left[\frac{1}{2}, a, a+1,-\frac{f}{\lambda}\right]-P^{\frac{n-1}{2}}{ }_{2} F_{1}\left[\frac{1}{2}, a, a+1,-\frac{f P^{n-3}}{\lambda}\right]\right\}
$$

The nature of the singularity can be characterized by the existence of radial null geodesics emerging from the singularity. The singularity is at least locally naked if there exist such geodesics and if no such geodesics exist it is a black hole. If the singularity is naked, then there exists a real and positive value of $X_{0}$ as a solution to the equation

$$
X_{0}=\lim _{t \rightarrow 0} \underset{r \rightarrow 0}{\stackrel{t}{r}}=\lim _{t \rightarrow 0} \underset{r \stackrel{d t}{d r}=\lim _{t \rightarrow 0} \frac{R^{\prime}}{\sqrt{1+f}}}{\stackrel{\frac{d}{\rightarrow} 0}{\rightarrow} 0}
$$

Define $\lambda_{0}=\lambda(0), \alpha_{0}=\alpha(0), f_{0}=f(0)$, and $Q=Q(X)=P(X, 0)$. Now from equation (9) it is seen that $\beta(0)=n-3$. We would denote $Q_{0}=Q\left(X_{0}\right)$, the equations (13) and (14) reduces to 


$$
X_{0}=\frac{2}{(n-1) \sqrt{\lambda_{0}}}\left\{{ }_{2} F_{1}\left[\frac{1}{2}, a, a+1,-\frac{f_{0}}{\lambda_{0}}\right]-Q_{0}^{\frac{n-1}{2}}{ }_{2} F_{1}\left[\frac{1}{2}, a, a+1,-\frac{f_{0} Q_{0}^{n-3}}{\lambda_{0}}\right]\right\}
$$

and

$$
\begin{aligned}
X_{0}= & \frac{1}{(n-3) \sqrt{1+f_{0}}}\left[\frac{1}{n-1}\left\{Q_{0}{ }_{2} F_{1}\left[\frac{1}{2}, a, a+1,-\frac{f_{0} Q_{0}^{n-3}}{\lambda_{0}}\right]-Q_{0}^{\frac{3-n}{2}}{ }_{2} F_{1}\left[\frac{1}{2}, a, a+1,-\frac{f_{0}}{\lambda_{0}}\right]\right\}\right. \\
& \left.\left\{\alpha_{0}(n-1)-2 n+6\right\} \sqrt{1+\frac{f_{0} Q_{0}^{n-3}}{\lambda_{0}}}+\frac{\alpha_{0} Q_{0}^{\frac{3-n}{2}} \sqrt{1+\frac{f_{0} Q_{0}^{n-3}}{\lambda_{0}}}}{\sqrt{1+\frac{f_{0}}{\lambda_{0}}}}+Q_{0}\left(n-3-\alpha_{0}\right)\right]
\end{aligned}
$$

\begin{tabular}{|c|c|c|c|c|c|c|c|c|c|c|}
\hline \multirow[t]{2}{*}{$f_{0}$} & \multirow[t]{2}{*}{$\lambda_{0}$} & \multirow[t]{2}{*}{$\alpha_{0}$} & \multicolumn{3}{|l|}{0} & \multicolumn{3}{|c|}{ Positive roots $\left(X_{0}\right)$} & \multirow[b]{2}{*}{$12 \mathrm{D}$} & \multirow[b]{2}{*}{$16 \mathrm{D}$} \\
\hline & & & $4 \mathrm{D}$ & $5 \mathrm{D}$ & $6 \mathrm{D}$ & $7 \mathrm{D}$ & $8 \mathrm{D}$ & $10 \mathrm{D}$ & & \\
\hline-.033 & 3.034 & $4 \quad .2$ & $\begin{array}{l}6.75507 \\
1.14476\end{array}$ & $\begin{array}{l}4.32936 \\
1.16321\end{array}$ & $\begin{array}{l}3.15991 \\
1.1879\end{array}$ & $\begin{array}{l}2.44524 \\
1.22434\end{array}$ & $\begin{array}{l}1.92883 \\
1.29065\end{array}$ & $\begin{array}{l}.669541 \\
.415056\end{array}$ & - & - \\
\hline-.1 & .333 & 3 & $\begin{array}{l}3.15662, \\
1.24003\end{array}$ & $\begin{array}{l}1.72737 \\
1.48602\end{array}$ & - & - & - & - & - & - \\
\hline-.301 & 1.302 & 2.001 & $\begin{array}{l}2.07773 \\
1.65625\end{array}$ & - & - & - & - & - & - & - \\
\hline-.5 & .51 & .001 & - & - & - & - & - & - & - & - \\
\hline .1 & .1 & 2 & $\begin{array}{c}1.667 \\
.806578\end{array}$ & $\begin{array}{c}1.1946 \\
.858211\end{array}$ & - & - & - & - & - & - \\
\hline .1 & .1 & 10 & .463679 & .465426 & .466489 & .466499 & .464947 & .45438 & .429651 & .35278 \\
\hline .1 & 1 & .2 & - & - & - & - & - & - & - & - \\
\hline .1 & 5 & 5 & - & - & - & - & - & - & - & - \\
\hline 1 & .001 & 2 & $\begin{array}{l}.996033, \\
.414324\end{array}$ & $\begin{array}{l}.968869, \\
.41436\end{array}$ & $\begin{array}{l}.91379 \\
.414412\end{array}$ & $\begin{array}{l}.848544 \\
.414489\end{array}$ & $\begin{array}{l}.783787, \\
.414605\end{array}$ & $\begin{array}{l}.669541, \\
.415056\end{array}$ & $\begin{array}{l}.577489, \\
.416214\end{array}$ & - \\
\hline 10 & 001. & .2 & $\begin{array}{l}.316014, \\
.275327\end{array}$ & $\begin{array}{l}.309739 \\
.275856\end{array}$ & - & - & - & - & - & - \\
\hline 10 & .1 & 5 & .089435 & .089441 & .089435 & .089409 & .08935 & .089061 & .088347 & .084352 \\
\hline 10 & 1 & 3 & .128192 & .128966 & $\begin{array}{l}.19718, \\
.41436\end{array}$ & $\begin{array}{l}.169063, \\
.414412 \\
\end{array}$ & $\begin{array}{l}.142103, \\
.414489 \\
\end{array}$ & - & - & - \\
\hline
\end{tabular}

TABLE: Positive values of $X_{0}$ by eliminating $Q_{0}$ from equations (15) and (16) for different values of the parameters $f_{0}, \lambda_{0}$ and $\alpha_{0}$ in various dimensions.

From above table we conclude the following results: 
(i) For same value of the parameters $\left(f_{0}, \lambda_{0}, \alpha_{0}\right)$ the possibility of positive real root is more in $4 \mathrm{D}$ and then it decreases with increase in dimensions.

(ii) If $\lambda_{0}$ is positive but close to zero then it is possible to have a naked singularity.

(iii) For same values of $f_{0}$ and $\lambda_{0}$, the naked singularity in higher dimensions will less probable as we increase the value the parameter $\alpha_{0}$.

The strength of singularity [15], which is the measure of its destructive capacity, is the most important feature. A singularity is gravitationally strong or simply strong if it destroys by crushing or stretching any objects that fall into it. Following Clarke and Krolak [16], we consider the null geodesics affinely parameterized by $\mu$ and terminating at shell focusing singularity $r=t=\mu=0$. Then it would be a strong curvature singularity as defined by Tipler [17] if

$$
\lim _{\mu \rightarrow 0} \mu^{2} \psi=\lim _{\mu \rightarrow 0} \mu^{2} R_{a b} K^{a} K^{b}>0
$$

where $R_{a b}$ is the Ricci tensor. It is widely believed that a space-time does not admit analytic extension through a singularity if it is a strong curvature singularity in the above sense. Now equation (17) can be expressed as

$$
\lim _{\mu \rightarrow 0} \mu^{2} \psi=\lim _{\mu \rightarrow 0} \frac{(n-2) F^{\prime}}{2 r^{n-4} P^{n-4} R^{\prime}}\left(\frac{\mu K^{t}}{R}\right)^{2}
$$

Using L'Hospital's rule and using equations (9) and (10), the equation (18) can be written as

$$
\lim _{\mu \rightarrow 0} \mu^{2} \psi=\frac{2(n-2)(n-3) \lambda_{0} Q_{0} X_{0}\left(\lambda_{0}+f_{0} Q_{0}^{n-3}\right) \sqrt{1+f_{0}}}{\left[(n-3) \lambda_{0} X_{0} \sqrt{1+f_{0}}-(n-3) \lambda_{0} Q_{0}-\alpha_{0} f_{0} Q_{0}^{n-2}\right]^{2}}>0
$$

Thus along the radial null geodesics, the strong curvature condition is satisfied and hence it is a strong curvature singularity.

Finally, we conclude that formation of naked singularity will be less probable as we increase the dimension of space-time.

\section{Acknowledgement:}

The authors are thankful to the members of Relativity and Cosmology Research Centre, Department of Physics, Jadavpur University for helpful discussion. One of the authors (U.D) is thankful to CSIR (Govt. of India) for awarding a Junior Research Fellowship.

\section{References:}

[1] S.W. Hawking and G.F.R. Ellis, The large scale structure of space-time (Cambridge. Univ. Press, Cambridge, England, 1973).

[2] R. Penrose, Riv. Nuovo Cimento 1252 (1969); in General Relativity, an Einstein Centenary Volume, edited by S.W. Hawking and W. Israel (Camb. Univ. Press, Cambridge, 1979).

[3] For recent reviews, see, e.g. P.S. Joshi, Pramana 55529 (2000); C. Gundlach, Living Rev. Rel. 24 (1999); A. Krolak, Prog. Theo. Phys. Suppl. 13645 (1999); R. Penrose, in Black holes and relativistic stars, ed. R. M. Wald (Univ. of Chicago Press, 1998); T.P.Singh, gr-qc/9805066; J. P. S. Lemos, Phys. Lett. A 158271 (1991); Phys. Rev. Lett. 681447 (1992); A. IIha and J. P. S. Lemos, Phys. Rev. D 551788 (1997); A. IIha, A. Kleber and J. P. S. Lemos, J. Math. Phys. 403509 (1999).

[4] P.S. Joshi, Global Aspects in Gravitation and Cosmology (Oxford Univ. Press, Oxford, $1993)$.

[5] C.J.S. Clarke, Class. Quantum Grav. 101375 (1993);T.P. Singh, J. Astrophys. Astron. 20 
$221(1999)$.

[6] F.C. Mena, R. Tavakol and P.S. Joshi, Phys. Rev. D 62044001 (2000).

[7] A. Benerjee, A. Sil and S. Chatterjee, Astrophys. J. 422681 (1994); A. Sil and S. Chatterjee, Gen. Rel. Grav. 26999 (1994); S. Chatterjee, A. Banerjee and B. Bhui, Phys. Lett. A 14991 (1990).

[8] S. G. Ghosh and A. Beesham, Phys. Rev. D 64124005 (2001); Class. Quantum Grav. 17 4959 (2000).

[9] S. G. Ghosh and A. Banerjee, Int. J. Mod. Phys. D,(2002) (accepted),gr-qc/0212067 (2002).

[10] P.S. Joshi, N. Dadhich and R. Maartens, Phys. Rev. D 65 101501(R)(2002).

[11] A. Banerjee, U. Debnath and S. Chakraborty, gr-qc/0211099 (2002) .

[12] U. Debnath and S. Chakraborty, gr-qc/0211102 (2002) .

[13] R. Goswami and P.S. Joshi, gr-qc/02112097 (2002) .

[14] I. H. Dwivedi and P. S. Joshi, Class. Quantum Grav. 9 L69 (1992).

[15] F. J. Tipler, Phys. Lett. A 648 (1987).

[16] C. J. S. Clarke and A. Krolak, J. Geom. Phys. 2127 (1986).

[17] F. J. Tipler, C. J. S. Clarke and G. F. R. Ellis, General Relativity and Gravitation ed. A Held (New York, Plenum) 1980. 